Louisiana State University

LSU Digital Commons

$5-7-2008$

\title{
Can megavoltage computed tomography reduce proton range uncertainties in treatment plans for patients with large metal implants?
}

\author{
Wayne D. Newhauser \\ University of Texas Health Science Center at Houston \\ Annelise Giebeler \\ University of Texas Health Science Center at Houston \\ Katja M. Langen \\ M. D. Anderson Cancer Center Orlando \\ Dragan Mirkovic \\ University of Texas Health Science Center at Houston \\ Radhe Mohan \\ University of Texas Health Science Center at Houston
}

Follow this and additional works at: https://digitalcommons.Isu.edu/physics_astronomy_pubs

\section{Recommended Citation}

Newhauser, W., Giebeler, A., Langen, K., Mirkovic, D., \& Mohan, R. (2008). Can megavoltage computed tomography reduce proton range uncertainties in treatment plans for patients with large metal implants?. Physics in Medicine and Biology, 53 (9), 2327-2344. https://doi.org/10.1088/0031-9155/53/9/009 


\title{
Can megavoltage computed tomography reduce proton range uncertainties in treatment plans for patients with large metal implants?
}

\author{
Wayne D Newhauser ${ }^{1,2}$, Annelise Giebeler ${ }^{1,2}$, Katja M Langen ${ }^{3}$, Dragan Mirkovic ${ }^{1,2}$, and \\ Radhe Mohan ${ }^{1,2}$ \\ ${ }^{1}$ Department of Radiation Physics, University of Texas M. D. Anderson Cancer Center, 1515 \\ Holcombe Boulevard, Unit 94, Houston, TX 77030, USA \\ ${ }^{2}$ University of Texas Graduate School of Biomedical Sciences, 6767 Bertner, Houston, TX 77030, \\ USA \\ ${ }^{3}$ M D Anderson Cancer Center Orlando, 1400 S Orange Avenue, Orlando, FL 32806, USA
}

\begin{abstract}
Treatment planning calculations for proton therapy require an accurate knowledge of radiological path length, or range, to the distal edge of the target volume. In most cases, the range may be calculated with sufficient accuracy using kilovoltage (kV) computed tomography (CT) images. However, metal implants such as hip prostheses can cause severe streak artifacts that lead to large uncertainties in proton range. The purposes of this study were to quantify streak-related range errors and to determine if they could be avoided by using artifact-free megavoltage (MV) CT images in treatment planning. Proton treatment plans were prepared for a rigid, heterogeneous phantom and for a prostate cancer patient with a metal hip prosthesis using corrected and uncorrected $\mathrm{kVCT}$ images alone, uncorrected MVCT images and a combination of registered MVCT and kVCT images (the hybrid approach). Streak-induced range errors of 5-12 mm were present in the uncorrected kVCT-based patient plan. Correcting the streaks by manually assigning estimated true Hounsfield units improved the range accuracy. In a rigid heterogeneous phantom, the implant-related range uncertainty was estimated at $<3 \mathrm{~mm}$ for both the corrected kVCT-based plan and the uncorrected MVCT-based plan. The hybrid planning approach yielded the best overall result. In this approach, the kVCT images provided good delineation of soft tissues due to high-contrast resolution, and the streak-free MVCT images provided smaller range uncertainties because they did not require artifact correction.
\end{abstract}

\section{Introduction}

Most external-beam radiotherapy treatments are planned primarily on the basis of threedimensional kilovoltage computed tomography (kVCT) scans. The images are typically reconstructed from measurements of the photon transmission through the patient (Hoheisel 
2006). Metallic implants, e.g. hip prostheses and dental implants, can severely reduce the transmission of photons, leading to a saturation of the CT image in the region of the implant and to streaking artifacts that may extend far from the implant (Jakel and Reiss 2007). These artifacts can result in errors in the dose distribution calculated by the treatment planning system. Specifically, for photon beam radiotherapy, the planning system converts CT images from Hounsfield units (HU) to electron densities; for proton therapy, the CT images are converted to relative linear proton stopping powers. In photon beam therapy, the streak artifacts are of lesser concern because they produce relatively small errors in the dose predicted by the planning system, mainly because the therapeutic photon dose is attenuated exponentially with depth in the patient. In stark contrast, streak artifacts are of greater concern in proton therapy because they translate into proton range errors which then may result in severe underdosing of the target volume (Wei et al 2006) or overdosing of normal tissue distal to the target. In the special case of patch-field treatments, a range error in the patch beam can result in severe under- or overdosing of the target volume. Beam orientations should be selected to avoid passing through or near to the metal implant, if possible (Jakel and Reiss 2007). Until now, the CT numbers of image pixels within artifact regions in KVCT images have been manually substituted with assumed values or alternatively, computer algorithms were applied before or after image reconstruction (Wang et al 2000, De Man et al 2001, Wei et al 2006, Zhang et al 2006).

Several investigations have shown that it is possible to substantially reduce image artifacts using computed tomography with megavoltage photons (MVCT) (Simpson et al 1982, Swindell et al 1983, Brahme et al 1987), which are not severely attenuated in metal implants. However, the soft tissue contrast and spatial resolution of MVCT scans are inferior to those of $\mathrm{kVCT}$ scans. MVCT has been used for photon treatment planning dose recalculations (Langen et al 2005). However, it is not known whether MVCT can be used in proton treatment planning to manage the range uncertainties associated with $\mathrm{CT}$ artifacts.

The purpose of our study was to determine whether proton beam range uncertainties can be reduced by using artifact-free MVCT images in the treatment planning process. We accomplished this by performing a comparative treatment planning study in which a patient with a metallic hip prosthesis was to receive passively spread proton therapy beams.

\section{Methods and materials}

A hybrid approach to treatment planning was evaluated in which both KVCT and MVCT images were utilized. To determine whether the hybrid planning approach reduced proton range uncertainties in the patient, we first had to obtain baseline data to establish the range accuracy of the MVCT-based treatment planning approach in a phantom. The phantom and patient studies both used the same CT scanning techniques (section 2.1) and treatment planning methods (section 2.2). The baseline data to establish the range accuracy in the MVCT method were generated using rigid heterogeneous phantoms (sections 2.1 and 2.3). Finally, section 2.4 describes the methods used to test the central hypothesis of this work, i.e. whether MVCT images can be used to reduce implant-related range uncertainties in a patient. 


\subsection{CT image acquisition}

The first study was performed in a rigid phantom to avoid organ motion and deformation, the effects of which might mask differences in the proton range computations. We constructed a phantom which is shown in figure 1 . It consisted of a plastic container $(30 \mathrm{~cm}$ $\times 15 \mathrm{~cm} \times 7 \mathrm{~cm}$ ) filled with rice flour that contained six cylindrical plugs, each of approximately $2.7 \mathrm{~cm}$ diameter and $7 \mathrm{~cm}$ height, which were distributed in the phantom with their axes parallel to the direction of travel of the CT couch. The plugs were each composed of a different material (cortical bone substitute CB2, $\rho=1.56 \mathrm{~g} \mathrm{~cm}^{-3}$; lung equivalent, $\rho=$ $0.3 \mathrm{~g} \mathrm{~cm}^{-3}$; solid water, $\rho=1.015 \mathrm{~g} \mathrm{~cm}^{-3}$; adipose tissue substitute, $\rho=0.92 \mathrm{~g} \mathrm{~cm}^{-3}$ and copper, $\left.\rho=8.96 \mathrm{~g} \mathrm{~cm}^{-3}\right)$. An MVCT scan of this phantom was done using a TomoTherapy Hi-ART II unit (TomoTherapy Incorporated, Madison, WI, USA) and immediately afterward the phantom was imaged using a Picker PQ2000 kVCT scanner (Picker, Highland Heights, OH, USA). The copper plug was then carefully removed to avoid disturbing the other plugs and the void was filled with rice flour. A second kVCT scan of this phantom was then obtained to serve as an artifact-free reference scan.

To investigate the use of MVCT and kVCT images for treatment planning, we used images of a patient treated for prostate cancer with a TomoTherapy unit. The kVCT images were obtained for the purpose of treatment planning and daily MVCT images were obtained for daily alignment. This patient was first imaged using the KVCT scanner with a helical scan and reconstructed using a $3 \mathrm{~mm}$ slice thickness with a reconstruction matrix of $512 \times 512$ pixels. (The $\mathrm{kV}$ scan was exported in a proprietary data format at full resolution, and then imported into the tomotherapy planning system. The images were then exported in DICOM format from the tomotherapy planning system, which downsampled the data to $256 \times 256$ pixels. While downsampling was not desired, the final images still provided adequate spatial resolution for the purposes of this study.) Within 1 week of the kVCT scanning, the patient was imaged using the MV imaging capabilities of the tomotherapy unit (Mackie et al 2003, Hoheisel 2006). This unit uses a $6 \mathrm{MV}$ accelerator to generate the imaging beam; however, during image acquisition the nominal energy of the incident electron beam is reduced to 3.5 $\mathrm{MeV}$ (Jeraj et al 2004). A xenon ion-chamber array is mounted opposite the accelerator and is used to acquire the imaging data. The gantry continuously rotates while the patient is translated through the imaging plane. The geometry of the MVCT imaging system is hence identical to that of a single-slice helical CT scanner. The nominal slice thickness of the MVCT scan was $4 \mathrm{~mm}$, with a reconstruction matrix of $512 \times 512$ pixels. Figure 2 presents representative transverse $\mathrm{CT}$ images that show the hip prosthesis, prostate and artifacts.

\subsection{Calibration of treatment planning system for proton dose calculations using KVCT and MVCT patient images}

To investigate the use of MVCT in proton therapy treatment planning, we performed measurements to relate the HU values from the $\mathrm{kVCT}$ and MVCT scanners to proton linear stopping power ratios. Specifically, we used the CT scanners to measure the HU values in a commercial CT calibration phantom (RMI 467, Gammex RMI, Middleton, WI, USA). Langen et al (2005) described the phantom and its application to MVCT calibrations for photon therapy. Table 1 lists the phantom material properties and the measured HU values for the CT calibration measurements. 
For this study, however, we used the stoichiometric method (Schneider et al 1996) to calibrate the scanners for proton therapy. For the reader's convenience, a brief review of that process in given here. First, the influence of the photon spectrum on HU values was taken into account. To accomplish this, we determined the values of coefficients that characterize the relative contributions to the total linear attenuation coefficient from the photoelectric effect $\left(K^{\mathrm{ph}}\right)$, coherent scattering $\left(K^{\mathrm{co}}\right)$ and Compton scattering $\left(K^{\mathrm{KN}}\right)$. The total linear attenuation coefficient was calculated using

$$
\mu=\rho N_{g}\left(K^{p h} \tilde{Z}^{3.62}+K^{c o} \hat{Z}_{i}^{1.86}+K^{K N}\right),
$$

where $\rho$ is the mass density, $N_{g}$ is the number of electrons per mass and $\tilde{Z}$ and $\hat{Z}$ are the effective atomic numbers for photoelectric and coherent scattering interactions, respectively (Jackson and Hawkes 1981, Schneider et al 1996). Recall that the mean attenuation coefficient is dependent on the CT scan technique used, the phantom size and composition and variations in the photon spectrum with scanner model and age. The coefficients were deduced from a gradient search algorithm that minimized the difference between the theoretical and measured $\mathrm{HU}$ values. For the $\mathrm{kV}$ scanner, the search procedure yielded the following values: $K^{\mathrm{ph}}=1.10 \times 10^{-4}, K^{\mathrm{co}}=0$ and $K^{\mathrm{KN}}=4.762$. For the MV scanner, the values were $K^{\mathrm{ph}}=0, K^{\mathrm{co}}=0$ and $K^{\mathrm{KN}}=0.375$. Second, we calculated theoretical HU values according to

$$
H U=1000\left(\frac{\mu}{\mu_{\text {water }}}\right)
$$

and proton linear stopping powers from formulas from Zeigler and Biersack (1994) for a variety of tissues and tissue substitutes. A complete listing of the materials used was given by (Schneider et al 1996). Third, the correlation between the theoretical HU and the relative linear stopping power (RLSP) values was modeled with a function that comprises piecewise linear fits of the theoretical HU and RLSP values. Independent regression analyses were carried out for the regions from 0 to $900 \mathrm{HU}_{\mathrm{sc}}, 900$ to $1100 \mathrm{HU}_{\mathrm{sc}}$ and 1100 to $3000 \mathrm{HU}_{\mathrm{sc}}$. Finally, the HU versus RLSP curves for the kVCT and MVCT scanners were imported into the treatment planning system.

For the kVCT and MVCT scanners, respectively, figures 3 and 4 plot the stoichiometric calibration curves relating the proton RLSP to HU values for a variety of tissues and tissue substitute materials. The fitted tri-linear calibration curves agreed reasonably well with individual tissue data points. The characteristics of the $\mathrm{kV}$ and $\mathrm{MV}$ curves differed mainly in that the MV curve was nearly linear from 0 to $3000 \mathrm{HU}_{\mathrm{sc}}$, whereas the slope of the $\mathrm{kV}$ curve decreased at about $1000 \mathrm{HU}_{\mathrm{sc}}$. Another difference was that above $1300 \mathrm{HU}_{\mathrm{sc}}$, the $\mathrm{HU}$ value for any given material was systematically smaller on the MV curve than in the $\mathrm{kV}$ curve. The deviations between individual HU values and the fitted calibration curve were smaller for the MV scanner, particularly in the important soft-tissue interval from 800 to $1200 \mathrm{HU}_{\mathrm{sc}}$. This finding hints that MVCT scans may be more accurate than kVCT scans in calculating proton range. 


\subsection{Confirmation of range accuracy of MVCT-based phantom treatment plans}

In this section, we describe the baseline data that established the range accuracy in the MVCT planning method, a prerequisite step to the main goal of this study (see section 2.3). Two situations were considered: a streak-free plan and a plan that contained streak artifacts caused by the copper plug inside the phantom. In all plans, we selected field orientations such that the metal plug was not in the beam path. The best estimate of the 'true range', our basis of comparison, was estimated with the following procedure. First a plan (plan A) was created based on the streak-free $\mathrm{kVCT}$ scan of the heterogeneous phantom (i.e. the copper plug was not present). The fields from this treatment plan were then recalculated in a homogeneous water phantom using the 'verification plan' feature of the planning system. The beam range was then taken as the distance to the distal $90 \%$ isodose line. The streakfree $\mathrm{kVCT}$ approach was used to estimate the true range because the method has a solid theoretical basis (Schneider et al 1996); its accuracy has been verified with extensive measurements (Schneider et al 1996, Vanderstraeten et al 2007), and potentially confounding streak artifacts were avoided.

To determine how well the kVCT-based planning approach predicted the beam range when the scan contained corrected streak artifacts, we performed the following steps. Beams from plan A were copied to a second plan (plan B) that was identical except that the CT scan was done with the copper plug present, creating streak artifacts on the images. After streak corrections were applied (described in section 2.4), the dose distribution was recalculated as before, which included a newly calculated range compensator design. The proton beams passed through streaks but not through the metal plug. The ranges were extracted as before, using a water phantom verification plan. An identical procedure to that used for plan B was used to determine how well the MVCT-based plan predicted the beam range in the presence of streaks. This plan (plan C) utilized the MVCT scan of the phantom with the copper plug present.

The observed difference in the ranges derived from these phantom plans allowed us to evaluate the feasibility of using the MVCT-based planning approach for the study in section 2.4. The differences between the observed ranges (in plans B and C with the metal plug present) and the 'true' range (from plan A without the metal plug) are plotted in figures 5 and 6 as a function of the crossfield position in the phantom. For the kVCT-based plan, subtracting the range of 'plan A' from 'plan B' revealed that the error remaining after streak corrections led to an underestimation of the true range by $2-3 \mathrm{~mm}$. Regarding the MVCTbased plan, subtracting 'plan A' from 'plan C' revealed an overestimation of 2-3 mm. Hence, the range errors for the MVCT-based plan and the corrected-kVCT-based plan were virtually identical, at $2-3 \mathrm{~mm}$. This finding was confirmed by a more detailed, threedimensional analysis of the range differences in which $90 \%$ isodose surfaces were extracted from all three plans and pointwise distances between them were computed for the distal region of the beam. In this region, the similarity of the distance-to-agreement histograms, as shown in figure 7, confirm that the MVCT-based plan had virtually identical range accuracy to the manually corrected KVCT-based plan. For example, the maximum and the average distances between the corrected $\mathrm{kVCT}$ plan and the $\mathrm{kVCT}$ plan without the metal plug were $2.6 \mathrm{~mm}$ and $0.87 \mathrm{~mm}$, respectively, while the corresponding values for the MVCT and 
$\mathrm{kVCT}$ plan without the metal plug were $2.6 \mathrm{~mm}$ and $0.9 \mathrm{~mm}$, respectively. (It is important to note that this parity in range accuracy was achieved under best-case conditions for the kVCT-based approach. Specifically, we knew the exact topology and composition of the phantom plan and therefore we could make the streak corrections with little subjectivity and uncertainty.) Together, these findings for a simple phantom suggest that the true proton range in a complex patient anatomy with a metal implant can be estimated more reliably with the MVCT-based plan than with the KVCT-based plan. The reason for this difference in reliability is that the $\mathrm{kVCT}$ images require streak corrections, which can compromise range accuracy.

\subsection{Treatment planning strategies and evaluation methods for the prostate plans}

To investigate the dosimetric differences between treatment planning doses calculated using MVCT and kVCT scans, we explored two strategies. The first strategy was to plan the prostate treatment to the best of our ability using techniques that comprise the contemporary standard of care, i.e. using the KVCT images for structure delineation and dose calculations. Streak artifacts in the KVCT images were reduced by delineating the streaks and reassigning the HU values within the streak contours. The second strategy utilized the kVCT images for soft tissue delineation and MVCT images for delineation of the prosthesis and proton dose calculations. The remainder of this section describes these methods in detail.

The treatment plans were developed using the Eclipse treatment planning system (Varian Medical Systems, Palo Alto, CA, USA), which contains a pencil beam algorithm (Schaffner et al 1999) for proton beam calculations. The system was commissioned (Newhauser et al 2007) with beam data for the $250 \mathrm{MeV}$ proton therapy facility at M D Anderson Cancer Center (Smith et al 2002). The image editing and registration, dosimetric calculations and plan evaluation tasks were performed within the treatment planning system.

Streak artifacts were corrected in the kVCT image set according to the following approach. Regions containing large $\mathrm{HU}$ values were delineated using an automatic tool in the planning system. The tool created polygons that enveloped the regions that contained uncorrected HU values within a user-specified interval. The interval of uncorrected $\mathrm{HU}$ values was determined by an inspection of the artifacts. The boundaries of the automatically determined regions were then refined using a manual contouring tool in the planning system. Finally, the $\mathrm{HU}$ values inside the artifact boundaries were manually reassigned to a value of 1045 $\mathrm{HU}_{\mathrm{sc}}$. This value was selected based on an average of $\mathrm{HU}$ values sampled in artifact-free regions of tissue immediately superior to the artifact regions and verified by a HU sampled in the artifact-free tissue surrounding the contralateral hip. A similar procedure was performed to reassign $\mathrm{HU}$ values in the regions containing artifacts of low-HU values; these regions were also manually reassigned to $1045 \mathrm{HU}_{\mathrm{sc}}$. The result of this $\mathrm{CT}$ artifact editing procedure is shown in figure 8 .

The contouring, including delineation of the skin, prostate, seminal vesicles, rectum, bladder and hip prosthesis, was done using standard methods. We used a variation on the lateral field arrangement (Slater et al 2004), since it is now the most commonly used arrangement for proton treatments. As with the phantom study, we selected field orientations such that the prosthesis was not in the proton beam path. We used a lateral field through the normal hip 
and a posterior-oblique field orientation to avoid the prosthesis. The lateral margins, which expand the aperture in the final beam shaping collimator, were set to $1.2 \mathrm{~cm}$. The lateral margin $(L M)$ was calculated by the method of Moyers and Miller (2003) or

$$
L M=S U U+P,
$$

where $S U U$ is the setup uncertainty and $P$ is the $90-50 \%$ penumbral width. In this work, SUU was taken as $0.5 \mathrm{~cm}$ (Moyers and Miller 2003) and $P$ was assigned $0.7 \mathrm{~cm}$ based on measurements of $225 \mathrm{MeV}$ treatment beams at our facility. The distal margins $(D M)$ were approximately $11 \mathrm{~mm}$ and were calculated using

$$
D M=\alpha \bullet R+\beta,
$$

where $a$ is the range uncertainty coefficient associated with uncertainties in the CT image reconstruction, $R$ is the water-equivalent beam penetration range in centimeters and $\beta$ accounts for range uncertainties due to all other effects (Moyers et al 2001, Moyers and Miller 2003). The range values were $21 \mathrm{~cm}$ for the lateral field and $23.5 \mathrm{~cm}$ for the right posterior-oblique (RPO) field. In this work, we used $\alpha=0.035$ and $\beta=0.3 \mathrm{~cm}$ based on the values from Moyers and Miller (2003). The proximal margins were adjusted so that each individual field provided proximal coverage of the target. The amount of smearing of the range compensator $(S)$, which decreases the range compensator thickness to maintain target volume coverage in the presence of targeting uncertainties (Urie et al 1984) was calculated as

$$
S=\left[(\kappa \bullet R)^{2}+(S U U)^{2}\right]^{1 / 2},
$$

where $\kappa$ is the smearing range factor, $R$ is the range as defined previously and $S U U$ is the setup uncertainty (Moyers and Miller 2003). The value of $\kappa$ was taken as $0.03 \mathrm{~cm}$ and that of SUU $0.5 \mathrm{~cm}$ (Urie et al 1984, Moyers et al 2001, Moyers and Miller 2003), yielding smear radii in the interval from 0.79 to $0.86 \mathrm{~cm}$. The right and left beams were weighted equally.

For the hybrid kVCT/MVCT-based plan, the images were first registered to one another using the automatic pixel-by-pixel image registration algorithm in the planning system. The registration was refined by applying manual translations and rotations to minimize differences in the location of the bony landmarks of the pelvis. To facilitate the visual comparison of individual CT slices, prior to the registration process we interpolated the $\mathrm{kV}$ and MV datasets to a common longitudinal slice thickness of $3 \mathrm{~mm}$. The image registration in the vicinity of the prostate was slightly (approximately $3 \mathrm{~mm}$ ) compromised by internal organ motion that occurred between the acquisition of the two CT scans. This motion was representative of interfractional motion (Litzenberg et al 2006) and provided a means of verifying that the target volume margins would be sufficient to provide coverage of the prostate during organ motion. The soft-tissue contours from the kVCT images were copied to the MVCT images. The prosthesis was contoured in the MVCT images, where it was more clearly resolved, along with the skin. The beam angles and weights for the hybrid plan were taken from the $\mathrm{kVCT}$ plan, and the apertures and range compensators were designed anew using the same margins and smear radius formulas that were used in the KVCT plan. 
Both plans were designed to provide a mean dose at isocenter of $75.6 \mathrm{~Gy}$. Table 2 summarizes the major treatment planning parameters for each of the treatment fields considered.

To assess the range uncertainties in the individual KVCT and MVCT proton treatment fields, we compared the maximum ranges of each beam in the kVCT and the MVCT plans. For this comparison, we created verification plans in a water phantom, i.e. we calculated the dose distribution from each treatment portal in a homogeneous cubic water phantom. The only beam parameter that was modified for the verification plans was the gantry angle, which was adjusted for the lateral-posterior field so that beam was normally incident on the water phantom. This adjustment was done only to simplify the comparison of the maximum beam penetrations in the water phantoms.

\section{Results}

The kVCT image set contained saturation and streak artifacts that are typical of those that arise in imaging patients with implanted hip prostheses who undergo radiotherapy for prostate cancer. However, as expected, these images had generally good soft-tissue resolution, which is essential for delineating the prostate and other organs. Also as expected, the MVCT data contained comparatively faint artifacts but exhibited poorer soft-tissue resolution (figure 8).

The uncorrected and corrected kV-based plans and kV/MV-based plans are shown in figure 8 , which reveals nearly identical target coverage. Recall that the plans were designed to provide identical target coverage, i.e. the treatment planning system automatically adjusted the maximum beam ranges and the range compensator designs for optimized target coverage. Therefore, the dose distributions relative to anatomy in these plans were almost identical-the differences were mainly manifested in the planning system's required proton beam range and compensator design. In essence, the planning system forced a redesign of the range compensator, which in turn forced the ranges to appear (in the planning system) to be virtually identical. Thus, the true range differences were not really apparent from the dose distributions in figure 8 . Therefore, the true range differences were deduced from differences in artifact-free water box verification plans.

In summary, the similarity in the dose distributions in the patient anatomy was expected, but this similarity masks underlying differences due to streak artifacts and their corrections.

However, when the treatment beams were applied to the water phantom, the latent differences in the plans were revealed. Because the water phantom was completely homogeneous and free of CT artifacts, differences in the dose distributions in the water phantom were entirely due to differences in the patient plans themselves. Figure 9(B) shows the two-dimensional dose distributions from an individual beam of the uncorrected kVCTbased plan when the treatment field was incident on a water phantom. The gantry angle was set to $270^{\circ}$ so that the beam axes were normal to the front surface of the water phantom.

The range errors introduced by the $\mathrm{CT}$ artifacts, the main subject of this paper, were estimated from differences in penetration depths of the RPO and lateral fields in the $\mathrm{kV}$ 
plan, the artifact-corrected $\mathrm{kV}$ plan and the hybrid $\mathrm{kV} / \mathrm{MV}$ plan. Figure 10 plots differences between calculated ranges $(\Delta R)$ for the RPO field from the verification plans as a function of the off-axis position $(y)$ in the anterior-posterior direction on the central-axis slice. The ranges $(R)$ correspond to the distance from the phantom surface to the distal $90 \%$ isodose line. As indicated by the negative values for $\Delta R$, the uncorrected kVCT-based plan systematically underestimated the required range by approximately $5-12 \mathrm{~mm}$ across the field, with typical values of approximately $7 \mathrm{~mm}$ (figure 10, fine dashed line), relative to the MVCT-based plan. The artifact corrections to the $\mathrm{kV}$-based plan reduced these systematic errors in range by about half (figure 10, solid curve). The remarkably similar shape of these two curves suggests that, in correcting the $\mathrm{kV}$ scan, the geometries of the artifact regions were delineated correctly. In addition, the nearly constant offset between the curves suggests that the relative proton stopping power values that were assigned to the artifact regions were too small (HU values were manually assigned to a value of $1045 \mathrm{HU}_{\mathrm{sc}}$, corresponding to a RLSP value of 1.035). Last, the difference between the ranges in the corrected and uncorrected kVCT-based plans (figure 10, heavy dashed curve) ranged from about 3 to 6 $\mathrm{mm}$, providing a lower bound on the range uncertainty due to artifacts in the uncorrected $\mathrm{kVCT}$-based plan. Note that figure 10 plots range data for the $y$ interval from -2 to $6 \mathrm{~cm}$, i.e. we excluded small regions near the periphery of the field. Those regions were excluded because small geometrical field-alignment imperfections in the planning process, which are not important to this study, produced large apparent range differences at the field edges. These findings were confirmed by analysis of signed distances between $90 \%$ isodose surfaces for an anatomical volume of RPO field shown in figure 11. The sign here was assigned according to the sign of the inner product between the distance vector and the beam direction ( $x$-axis). The histograms show that both the kVCT plan and the corrected $\mathrm{kVCT}$ plan underestimated the beam range when compared to the MVCT-based plan.

A similar comparison of ranges from the verification plans for the left lateral (LL) field is shown in figure 12. The small $\Delta R$ values between the uncorrected and corrected kVCTbased plans reflect the minimal amount of visible streaking present in the beam path (see figure 2(B)). However, mostly the positive $\Delta R$ values indicate that the streak-corrected $\mathrm{kV}$ plan systematically overestimated the required beam range with respect to the MVCT-based plan, by as much as $12 \mathrm{~mm}$. This surprising result suggests that HU errors caused by implanted objects may be more pervasive and difficult to detect visually than previously appreciated. One possible explanation for this observation is that range small errors are accumulated when the proton beam path runs along the streaks. The compensator thickness for ray lines that mainly pass through high-HU streaks will thus be too thin. In addition, smearing of the range compensator design makes the device yet thinner, compounding the overshoot. Thus, the range of the protons in the patient is increased in regions of the field where the proton trajectories are approximately parallel to the streak artifacts. A corresponding and similar effect would occur for regions containing low-HU streaks.

\section{Discussion}

In proton treatment planning for a patient with prostate cancer who had a large metal hip prosthesis, the use of MVCT images for dose calculations reduced uncertainties in proton range relative to the uncertainties in calculations done using uncorrected $\mathrm{kVCT}$ images. The 
improvement in range accuracy was possible because the MVCT images contained only very faint streak artifacts. In contrast, the metal implants caused severe streak artifacts in the kVCT images that necessitated corrections, and the corrections contributed to the uncertainty in proton range. The streak correction method worked well in the case of a simple heterogeneous phantom, yielding an uncertainty of $\pm 3 \mathrm{~mm}$ in proton range due to imperfections in the streak correction. The corresponding range uncertainty in the prostate cancer treatment plan was much larger (from $+12 \mathrm{~mm}$ to $-8 \mathrm{~mm}$ ) because of the greater subjectivity involved in making the streak corrections. These results suggest that the management of streak artifacts in images of proton therapy patients with implants may be more important than previously appreciated. Also, our findings suggest that using MVCT images for proton dose calculations may provide a feasible alternative to manually correcting artifacts in $\mathrm{kVCT}$ images.

The kVCT-based plan for the patient systematically overestimated the required beam range for the LL field (far from the prosthesis) and underestimated the range for the RPO field (near the prosthesis). Therefore, the application of a single additive or multiplicative range correction factor will apparently not provide the intended result in every case. It is also interesting that the range error in the $\mathrm{kVCT}$ prostate plan (with streak corrections) was larger than we assumed, suggesting that a value of $a \geq 0.05$ should be used in calculating the distal margin size.

These findings suggest that the hybrid planning approach, i.e. using registered MVCT and kVCT data, yielded the best overall result. The kVCT images provided good delineation of soft tissues because of their high-contrast resolution. The MVCT images allowed for a reduction in the proton beam range uncertainties because they did not require streak corrections. In addition, the metal prosthesis could be delineated by using the MVCT data; accurate delineation is sometimes important in choosing the beam angle. The hybrid planning approach reported here was accomplished in a straightforward manner using clinical systems that are already commercially available.

Our study demonstrated the usefulness of MVCT images in proton therapy treatment planning. Specifically, proton doses may be calculated without having to correct for artifacts in the CT images. In particular, implant-induced saturation and streaking artifacts in kVCT scans, which normally require subjective, laborious and manual editing, were avoided entirely by performing the treatment planning dose calculations using an artifact-free MVCT dataset. In addition, our findings provide new estimates of the range errors that are associated with artifacts from a metal prosthesis. Our values thus may be used as lower bounds for estimating, for example distal margins in other patients with hip prostheses undergoing proton therapy for prostate cancer.

Our study had several limitations. First, the organ motion that occurred between the kVCT and MVCT sessions limited our ability to quantify the reduction in range uncertainties in the patient. In particular, tissue displacements may have slightly reduced or amplified the range uncertainties from CT artifacts, the main focus of this work. However, these displacements were very small and did not influence the general findings of our study. Second, our study considered only proton delivery to the pelvis; we do not yet know how useful MVCT will be 
for planning treatments at other sites. Despite these limitations, our results suggest that the $\mathrm{kV} / \mathrm{MV}$ hybrid approach would be useful for cases in which the proton beam must pass near to or through a metal implant, especially in cases of head and neck cancer in which dental fillings or surgical appliances cause severe streaking artifacts in $\mathrm{kV}$ scans. Additional investigation will be needed to determine whether this approach is suitable for clinical use.

In addition to avoiding streak artifacts, the MV-based approach yields advantages related to its greater dynamic range (i.e. higher saturation threshold) of relative linear stopping power values than that of $\mathrm{kV}$ scanning. Specifically, MVCT avoids saturation effects enabling more accurate delineation of the metal implant and thus theoretically enabling quantitative identification of the implant material. Alternatively, Coolens and Childs (2003) proposed to reduce saturation effects by using $\mathrm{kVCT}$ imaging with an extended dynamic range of $\mathrm{HU}$ values.

Our findings will allow us to take the next step in reducing range uncertainties and reducing the subjectivity and manual labor associated with correcting for implant-induced artifacts. Our results imply that MVCT has the potential to play an important role in proton therapy treatment planning, but this must be tested further and compared with other approaches, including streak-resistant kVCT reconstruction algorithms (McLean et al 2001, Yazdi et al 2005) and proton CT (Sadrozinski et al 2003).

\section{Acknowledgments}

This work was supported in part by a grant from Varian Medical Systems, Inc.

\section{References}

Brahme A, Lind B, Nafstadius P. Radiotherapeutic computed tomography with scanned photon beams. Int. J. Radiat. Oncol. Biol. Phys. 1987; 13:95-101. [PubMed: 3100482]

Coolens C, Childs PJ. Calibration of CT Hounsfield units for radiotherapy treatment planning of patients with metallic hip prostheses: the use of the extended CT-scale. Phys. Med. Biol. 2003; 48:1591-603. [PubMed: 12817940]

De Man B, Nuyts J, Dupont P, Marchal G, Suetens P. An iterative maximum-likelihood polychromatic algorithm for CT. IEEE Trans. Med. Imag. 2001; 20:999-1008.

Hoheisel M. Review of medical imaging with emphasis on X-ray detectors. Nucl. Instr. Meth. A. 2006; 563:215-24.

Jackson DF, Hawkes DJ. X-ray attenuation coefficients of elements and mixtures. Phys. Rep. 1981; 70:169-233.

Jakel O, Reiss P. The influence of metal artefacts on the range of ion beams. Phys. Med. Biol. 2007; 52:635-44. [PubMed: 17228110]

Jeraj R, Mackie TR, Balog J, Olivera G, Pearson D, Kapatoes J, Ruchala K, Reckwerdt P. Radiation characteristics of helical tomotherapy. Med. Phys. 2004; 31:396-404. [PubMed: 15000626]

Langen KM, Meeks SL, Poole DO, Wagner TH, Willoughby TR, Kupelian PA, Ruchala KJ, Haimerl J, Olivera GH. The use of megavoltage CT (MVCT) images for dose recomputations. Phys. Med. Biol. 2005; 50:4259-76. [PubMed: 16148392]

Litzenberg DW, Balter JM, Hadley SW, Sandler HM, Willoughby TR, Kupelian PA, Levine L. Influence of intrafraction motion on margins for prostate radiotherapy. Int. J. Radiat. Oncol. Biol. Phys. 2006; 65:548-53. [PubMed: 16545919]

Mackie TR, et al. Image guidance for precise conformal radiotherapy. Int. J. Radiat. Oncol. Biol. Phys. 2003; 56:89-105. [PubMed: 12694827] 
McLean D, Robinson J, Archer M. Artefact reduction on CT images of fossils to allow 3D visualisation. Radiat. Phys. Chem. 2001; 61:723-4.

Moyers MF, Miller DW. Range, range modulation, and field radius requirements for proton therapy of prostate cancer. Technol. Cancer Res. Treat. 2003; 2:445-7. [PubMed: 14529309]

Moyers MF, Miller DW, Bush DA, Slater JD. Methodologies and tools for proton beam design for lung tumors. Int. J. Radiat. Oncol. Biol. Phys. 2001; 49:1429-38. [PubMed: 11286851]

Newhauser W, Fontenot J, Zheng Y, Polf J, Titt U, Koch N, Zhang X, Mohan R. Monte Carlo simulations for configuring and testing an analytical proton therapy treatment planning system. Phys. Med. Biol. 2007; 52:4569-84. [PubMed: 17634651]

Sadrozinski HFW, et al. Issues in proton computed tomography. Nucl. Instr. Meth. A. 2003; 511:27581.

Schaffner B, Pedroni E, Lomax A. Dose calculation models for proton treatment planning using a dynamic beam delivery system: an attempt to include density heterogeneity effects in the analytical dose calculation. Phys. Med. Biol. 1999; 44:27-41. [PubMed: 10071873]

Schneider U, Pedroni E, Lomax A. The calibration of CT Hounsfield units for radiotherapy treatment planning. Phys. Med. Biol. 1996; 41:111-24. [PubMed: 8685250]

Simpson RG, Chen CT, Grubbs EA, Swindell W. A 4-MV CT scanner for radiation therapy: the prototype system. Med. Phys. 1982; 9:574-9. [PubMed: 7110089]

Slater JD, Rossi CJ Jr, Yonemoto LT, Bush DA, Jabola BR, Levy RP, Grove RI, Preston W, Slater JM. Proton therapy for prostate cancer: the initial Loma Linda University experience. Int. J. Radiat. Oncol. Biol. Phys. 2004; 59:348-52. [PubMed: 15145147]

Smith, A.; Newhauser, WD.; Latinkic, M.; Hay, A.; McMaken, B.; Styles, J.; Cox, J. The University of Texas M.D. Anderson Cancer Center proton therapy facility. 17th Int. Conf. Appl. Accel. Research and Industry; Denton, TX, USA. 12-16 November; New York: American Institute of Physics; 2002. p. 1073-6.

Swindell W, Simpson RG, Oleson JR, Chen CT, Grubbs EA. Computed tomography with a linear accelerator with radiotherapy applications. Med. Phys. 1983; 10:416-20. [PubMed: 6412044]

Urie M, Goitein M, Wagner M. Compensating for heterogeneities in proton radiation therapy. Phys. Med. Biol. 1984; 29:553-66. [PubMed: 6330772]

Vanderstraeten B, Chin PW, Fix M, Leal A, Mora G, Reynaert N, Seco J, Soukup M, Spezi E, De Neve W, Thierens H. Conversion of CT numbers into tissue parameters for Monte Carlo dose calculations: a multi-centre study. Phys. Med. Biol. 2007; 52:539-62. [PubMed: 17228104]

Wang G, Frei T, Vannier MW. Fast iterative algorithm for metal artifact reduction in X-ray CT. Academic Radiol. 2000; 7:607-14.

Wei J, Sandison GA, Hsi WC, Ringor M, Lu X. Dosimetric impact of a CT metal artefact suppression algorithm for proton, electron and photon therapies. Phys. Med. Biol. 2006; 51:5183-97. [PubMed: 17019032]

Yazdi M, Gingras L, Beaulieu L. An adaptive approach to metal artifact reduction in helical computed tomography for radiation therapy treatment planning: experimental and clinical studies. Int. J. Radiat. Oncol. Biol. Phys. 2005; 62:1224-31. [PubMed: 15927413]

Zeigler, JF.; Biersack, JP. TRIM-92 Transport of Ions in Matter. International Business Machines; Watson Laboratory, Yorktown: 1994.

Zhang Y, Zhang L, Zhu XR, Lee AK, Chambers M, Dong L. Reducing metal artifacts in cone-beam CT images by preprocessing projection data. Int. J. Radiat. Oncol. Biol. Phys. 2006; 67:924-32. [PubMed: 17161556] 

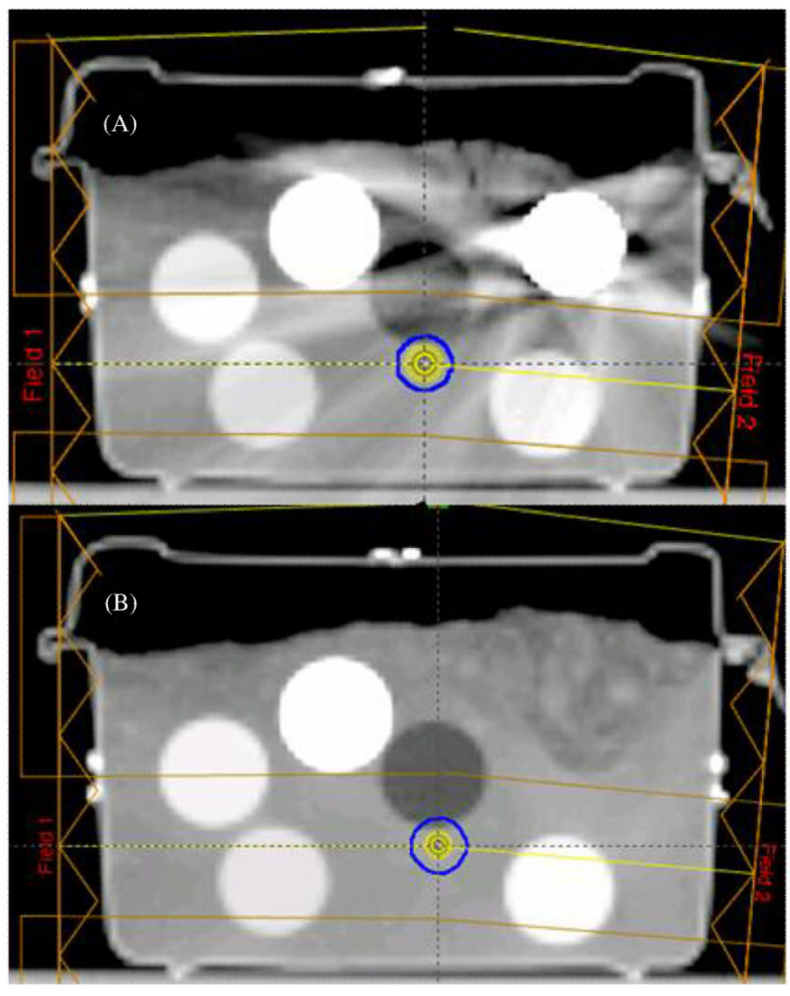

Figure 1.

(A) Lateral and lateral-oblique fields in the uncorrected kVCT treatment plan for the rice flour phantom with the copper plug present. (B) Lateral and lateral-oblique fields in the kVCT treatment plan for the rice flour phantom with the copper plug removed. The field edges are shown intersecting isocenter (at the center of the blue circle). 


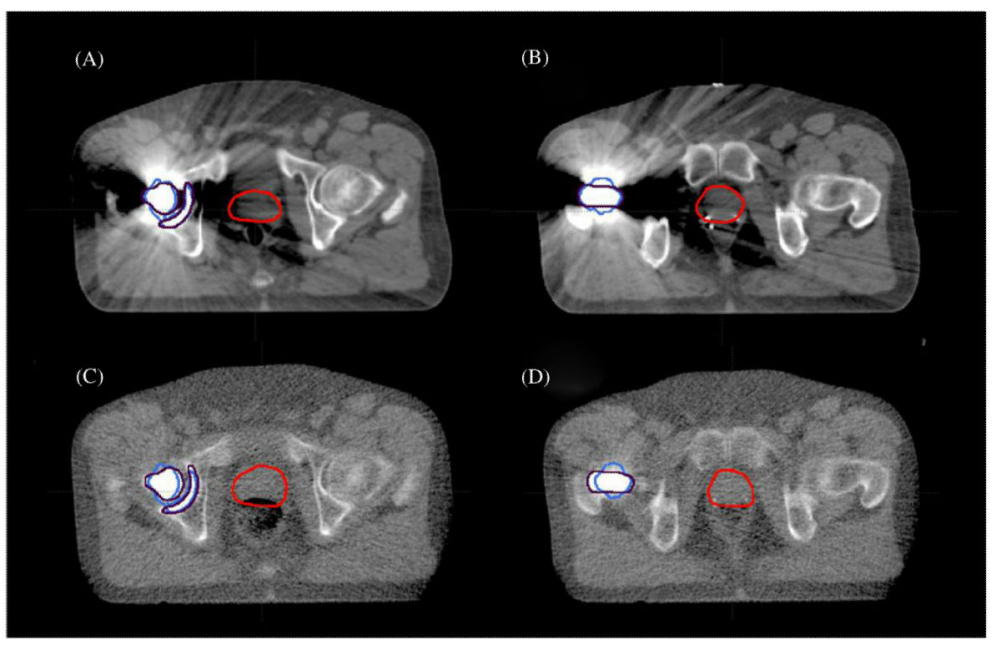

Figure 2.

(A, B) Representative kVCT images illustrating partial obscuring of boundaries of high- $Z$ objects. (C, D) Corresponding MVCT images showing clear boundaries. Blue and maroon lines represent prosthesis boundaries, respectively. Differences in the location of the prostate contours in the kVCT and MVCT images were due to anatomical changes between CT imaging sessions. Small differences in the shape of the prostate contours were introduced as they were interpolated onto the MVCT slices, which were offset slightly in the superiorinferior direction with respect to the KVCT slice positions. 


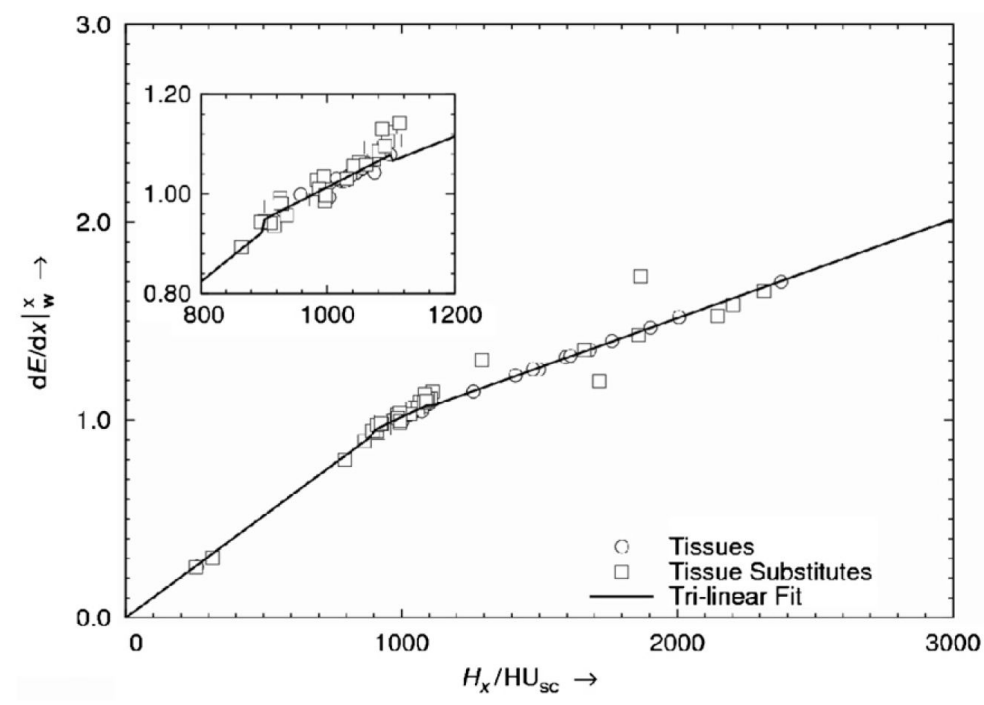

Figure 3.

Relative linear stopping power for protons $\left(\mathrm{d} E /\left.\mathrm{d} x\right|^{\mathrm{x}}{ }_{\mathrm{w}}\right)$ as a function of the scaled Hounsfield unit value $\left(H_{\mathrm{x}}\right.$, in units of $\left.\mathrm{HU}_{\mathrm{sc}}\right)$ in $\mathrm{kVCT}$, where $\mathrm{x}$ denotes a material of interest and $\mathrm{w}$ denotes water. 


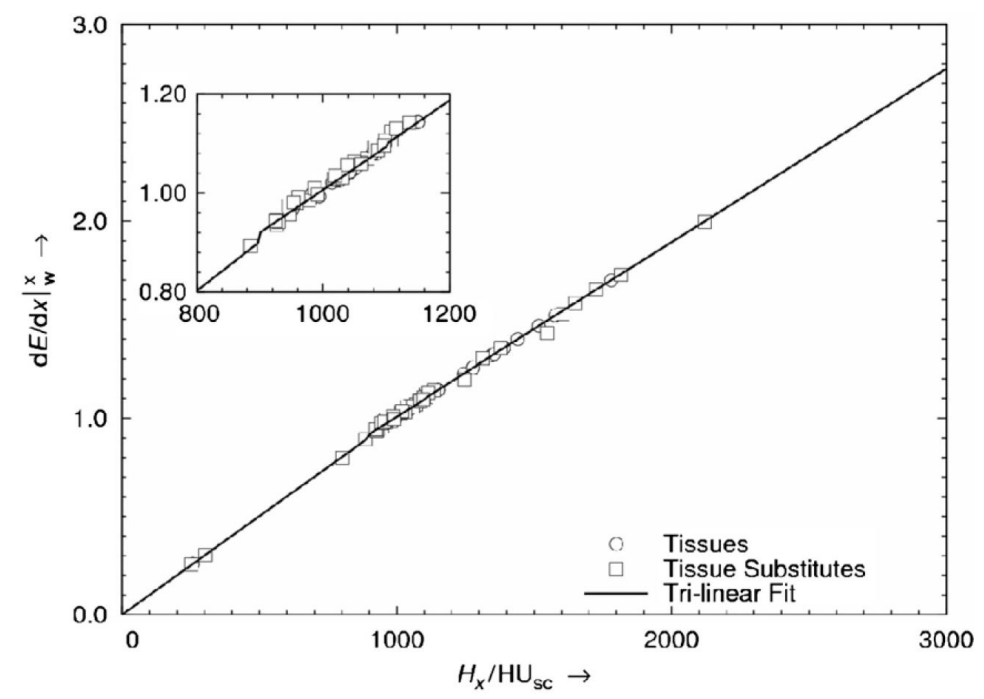

Figure 4.

Relative linear stopping power for protons $\left(\mathrm{d} E /\left.\mathrm{d} x\right|^{\mathrm{x}}{ }_{\mathrm{w}}\right)$ as a function of the scaled Hounsfield unit value $\left(H_{\mathrm{x}}\right.$, in units of $\left.\mathrm{HU}_{\mathrm{sc}}\right)$ in MVCT, where $\mathrm{x}$ denotes a material of interest and $\mathrm{w}$ denotes water. 


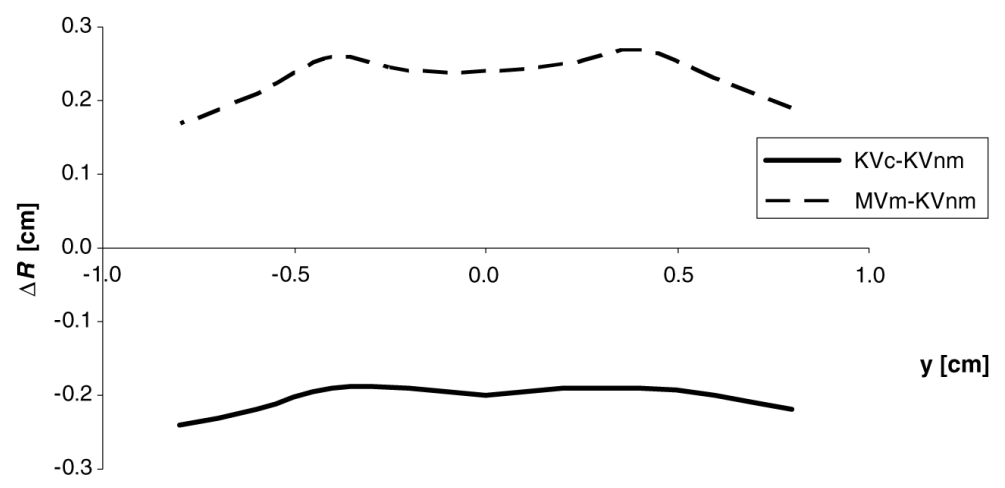

Figure 5.

Differences in penetration range $(\Delta R)$ between various planned ranges in the verification plans for the $\mathrm{kV}$ scan with metal artifacts corrected $(\mathrm{KVc})$, the $\mathrm{kV}$ scan with no metal $(\mathrm{KVnm})$ and the MV scan with metal $(\mathrm{MVm})$ as a function of the off-axis position $(y)$ in the anterior-posterior direction for the lateral field. 


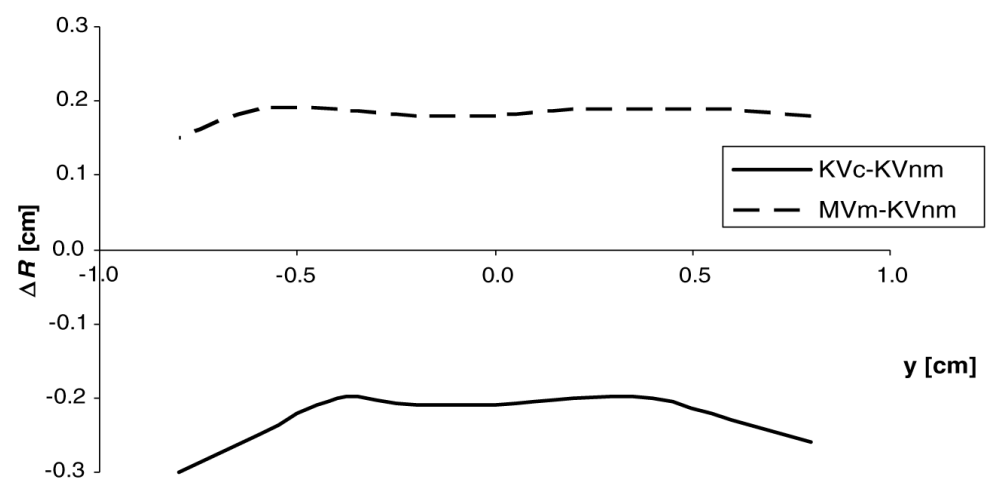

Figure 6.

Differences in penetration range $(\Delta R)$ between various planned ranges in the verification plans for the $\mathrm{kV}$ scan with metal artifacts corrected $(\mathrm{KVc})$, the $\mathrm{kV}$ scan with no metal $(\mathrm{KVnm})$ and the MV scan with metal $(\mathrm{MVm})$ as a function of the off-axis position $(y)$ in the anterior-posterior direction for the posterior-oblique field. 


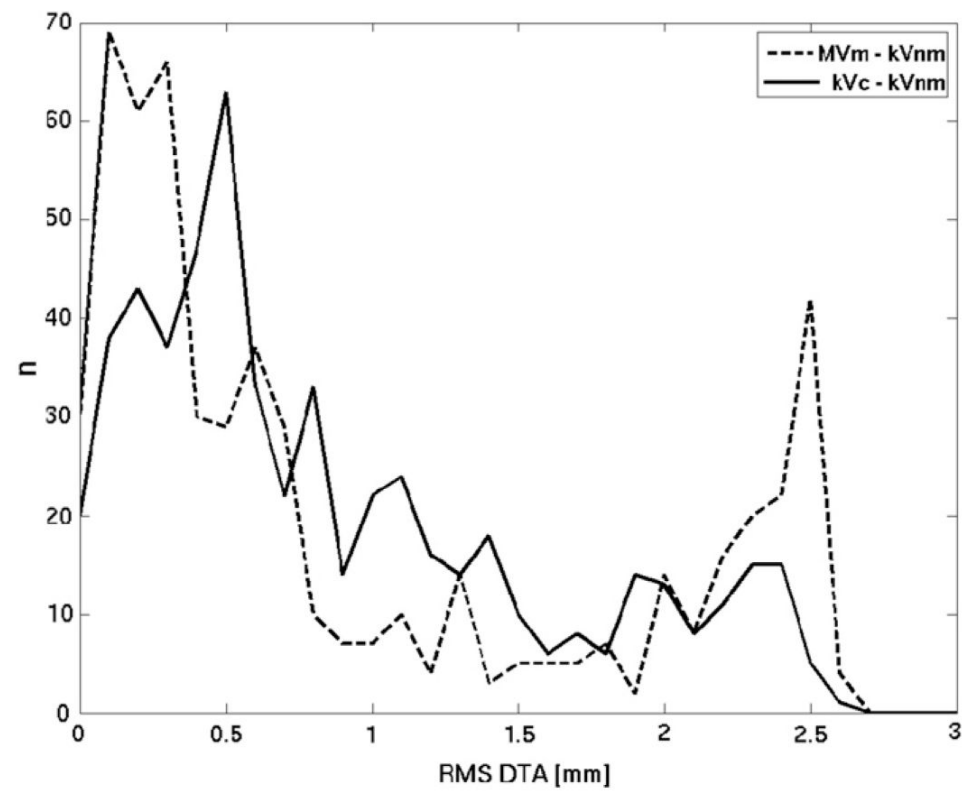

Figure 7.

Histogram of the frequency distribution of the rms distance to agreement (DTA) between the distal $90 \%$ isodose surfaces of the verification plans using the kVCT scan with metal artifacts corrected $(\mathrm{kVc})$, the $\mathrm{kVCT}$ scan with no metal $(\mathrm{kVnm})$ and the MVCT scan with metal (MVm) for the right posterior-oblique (RPO) field. The $\mathrm{kVc}$ and $\mathrm{MVm}$ isodose surfaces are uniformly close to the $\mathrm{kVnm}$ isodose surface with maximum distances of 2.63 and $2.56 \mathrm{~mm}$, respectively and average distances of 0.87 and $0.9 \mathrm{~mm}$, respectively. 


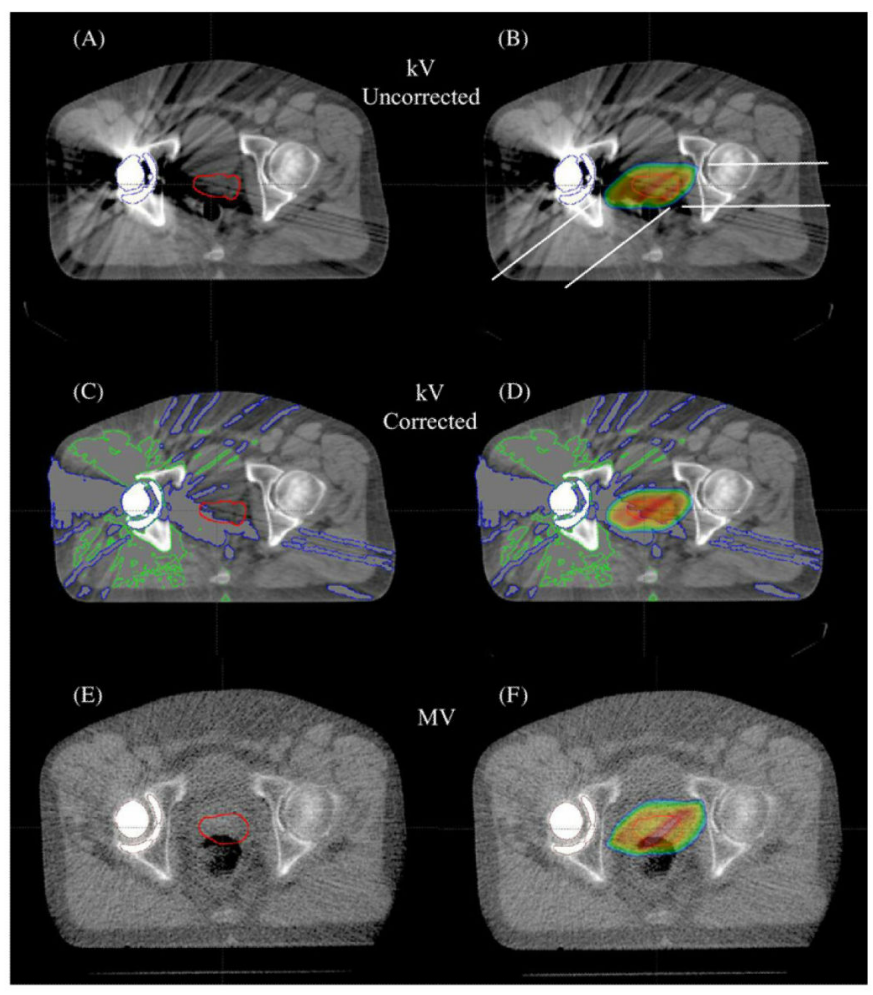

Figure 8.

KVCT and MVCT images of a prostate cancer radiotherapy patient with a metal hip prosthesis. (A) The kVCT image reveals image saturation within and adjacent to the prosthesis, as well as streaking artifacts emanating from the prosthesis and extending to the contralateral skin. (B) Calculated dose distribution using uncorrected kVCT images. White lines show lateral edges of fields. (C) Example kVCT image after correction for saturation and streaking artifacts. (D) Calculated dose distribution using corrected kVCT images. (E) MVCT image exhibiting poor soft tissue contrast resolution but free of saturation and streaking artifacts. (F) Calculated dose distribution using MVCT images. 


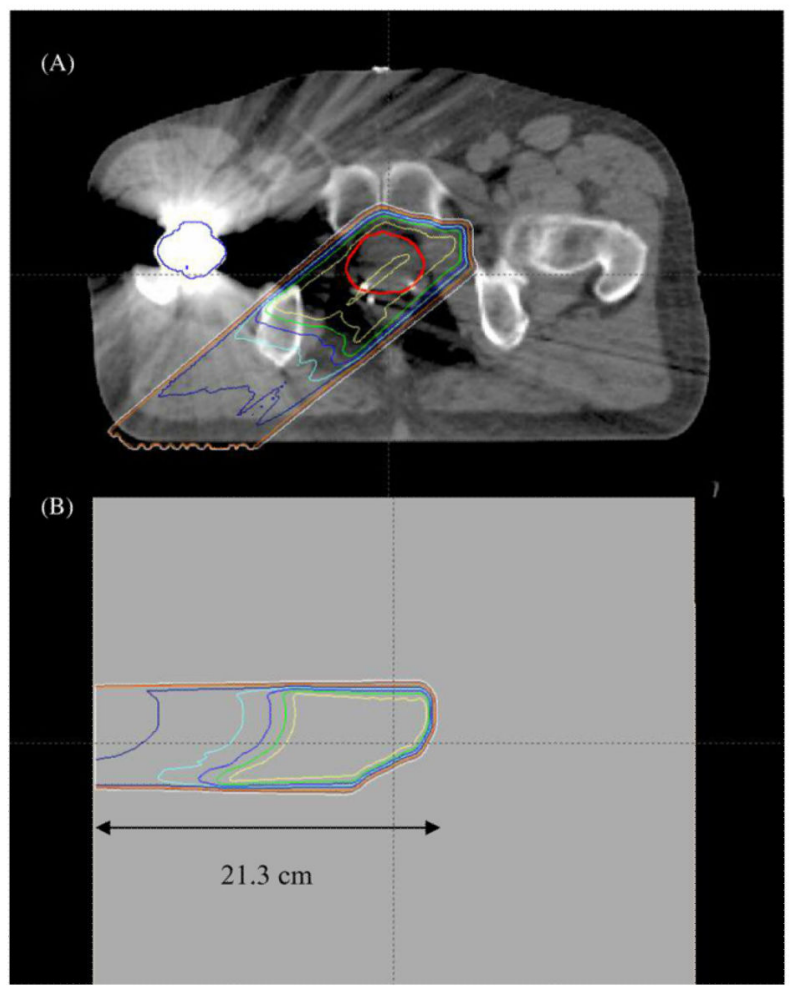

Figure 9.

(A) Dose distribution from the RPO field of the uncorrected kVCT treatment plan; the innermost (yellow) contours represent the $100 \%$ isodose level. (B) Dose distribution from the same RPO field when normally incident on a uniform water phantom. 


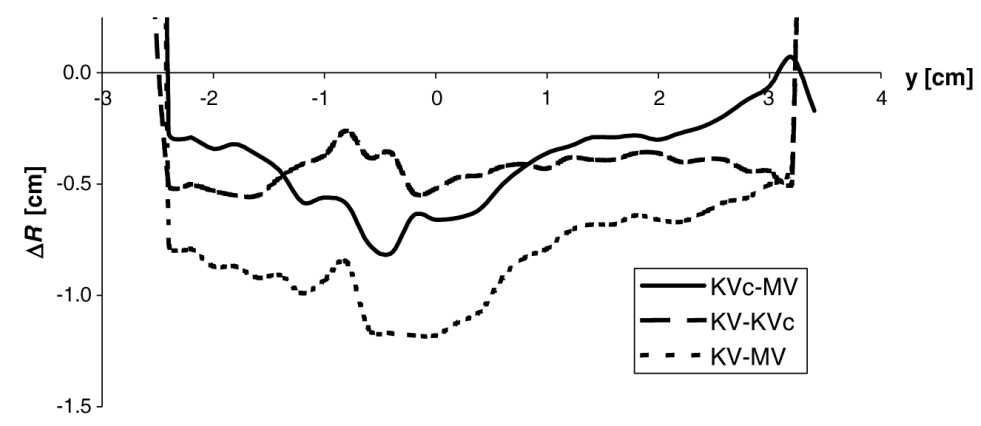

Figure 10.

Differences in penetration range $(\Delta R)$ between planned ranges in the verification plans as a function of the off-axis position $(y)$ in the anterior-posterior direction for the right posterioroblique field, incident normal to the water phantom. The uncorrected and corrected kVCTbased plans systematically underestimated the required range across the field as much as 12 $\mathrm{mm}$ and $8 \mathrm{~mm}$, respectively. Note that the large $\Delta R$ values between the uncorrected and corrected kVCT-based plans reflect the large amount of visible streaking present in the beam path (see figure 2(B)). 


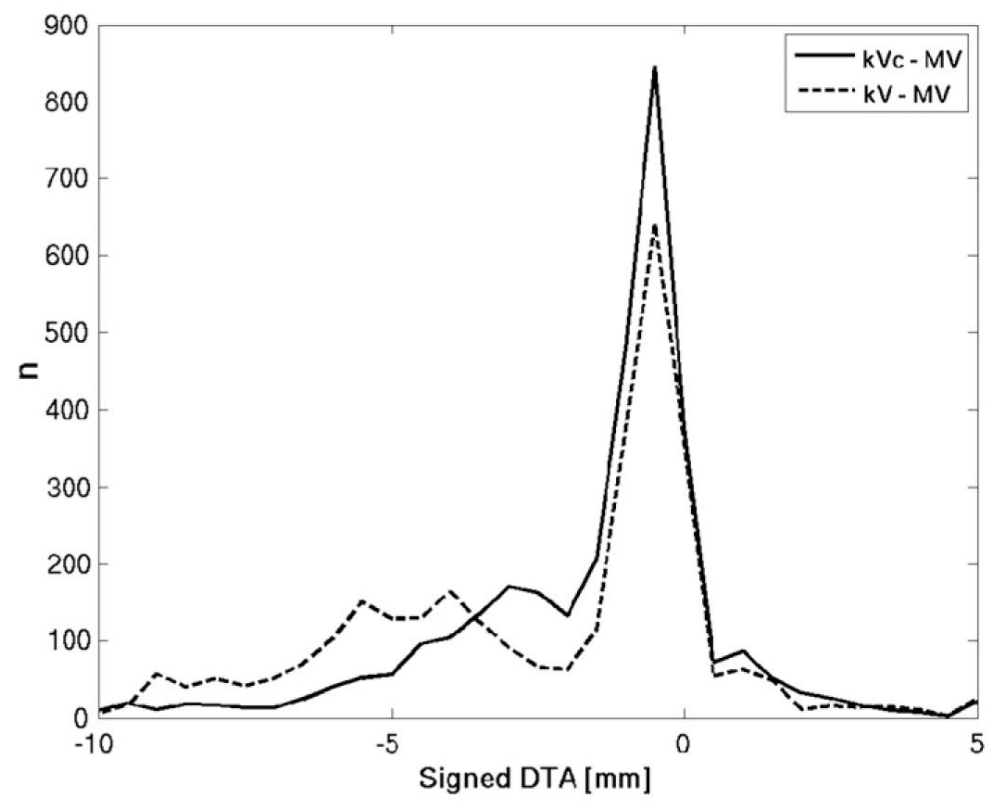

Figure 11.

Histograms of the signed distance to agreement (DTA) between the distal $90 \%$ isodose surfaces of the verification plans using the corrected kVCT scan and MVCT scan (kVc$\mathrm{MV}$ ), and between the uncorrected kVCT scan and the MVCT scan (kV - MV) for the right posterior-oblique (RPO) field. Both histograms exhibited a large number of negative values, indicating the underestimation of the range by plans based on $\mathrm{kVCT}$ and corrected $\mathrm{kVCT}$ scans. The frequency of large DTA values $(> \pm 4 \mathrm{~mm})$ was smaller when the kVCT scan was corrected, suggesting that the artifact corrections were only partially effective. 


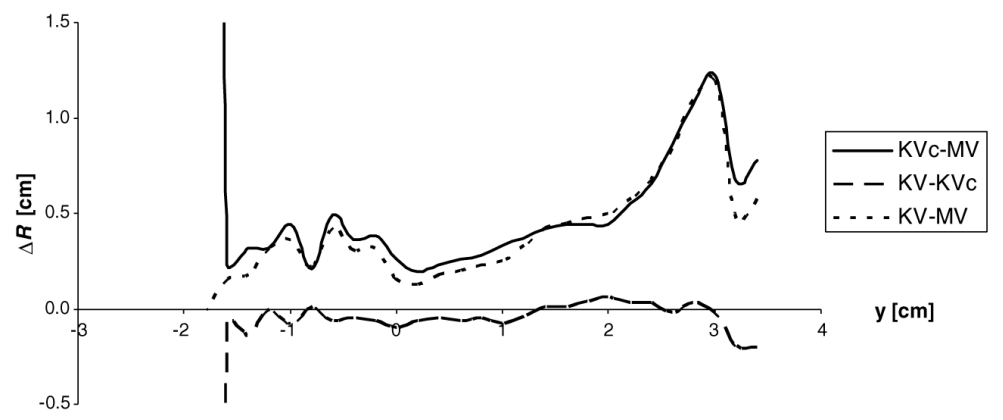

Figure 12.

Differences in penetration range $(\Delta R)$ between planned ranges in the verification plans as a function of the off-axis position $(y)$ in the anterior-posterior direction for the left lateral field. 


\section{Table 1}

Phantom properties and selected results for the calibration of the kilovoltage $(\mathrm{kV})$ and megavoltage (MV) computed tomography (CT) scanners, including phantom material, mass density $(\rho)$, scaled CT numbers from measurements $\left(H_{\mathrm{o}}\right)$ and theoretical predictions $\left(H_{\mathrm{p}}\right)$, and the absolute difference between the predicted and observed values. Note that the kVCT numbers for the metal inserts in the calibration phantom exceeded the dynamic range of the scanner and were therefore not reported.

\begin{tabular}{|c|c|c|c|c|c|c|c|}
\hline \multirow[b]{2}{*}{ Material } & \multirow[b]{2}{*}{$\rho\left(\mathrm{g} \mathrm{cm}^{-3}\right)$} & \multicolumn{3}{|r|}{$\mathbf{k V}$} & & MV \\
\hline & & $H_{\mathrm{p}}\left(\mathrm{HU}_{\mathrm{sc}}\right)$ & $H_{\mathrm{o}}\left(\mathrm{HU}_{\mathrm{sc}}\right)$ & $H_{\mathrm{p}}^{-} \boldsymbol{H}_{\mathbf{o}}\left(\mathbf{H U}_{\mathrm{sc}}\right)$ & $H_{\mathrm{p}}\left(\mathrm{HU}_{\mathrm{sc}}\right)$ & $H_{\mathrm{o}}\left(\mathrm{HU}_{\mathrm{sc}}\right)$ & $\boldsymbol{H}_{\mathrm{p}}^{-} \boldsymbol{H}_{\mathbf{o}}\left(\mathbf{H U}_{\mathrm{sc}}\right)$ \\
\hline Water (liquid) & 1.0 & 1000 & 1000 & 0.0 & 1000.0 & 1000 & 0.0 \\
\hline Cortical bone (SB3) & 1.823 & 2171 & 2158 & 12.7 & 1691.6 & 1642 & 49.6 \\
\hline Inner bone (IB) & 1.143 & 1170 & 1226 & 55.8 & 1103.0 & 1097 & 6.0 \\
\hline Liver (LV1) & 1.095 & 1074 & 1073 & 1.2 & 1064.2 & 1055 & 9.2 \\
\hline Breast (BR12) & 0.99 & 962 & 962 & 0.2 & 967.2 & 957 & 10.2 \\
\hline Lung (LN300) & 0.30 & 293 & 320 & 26.8 & 292.2 & 321 & 28.8 \\
\hline Lung (LN450) & 0.45 & 440 & 468 & 28.4 & 438.3 & 465 & 26.7 \\
\hline Adipose (AP6) & 0.92 & 883 & 914 & 30.8 & 897.4 & 927 & 29.6 \\
\hline Solid water & 1.015 & 996 & 1008 & 12.3 & 986.5 & 985 & 1.5 \\
\hline Brain (SR2) & 1.049 & 1027 & 1027 & 0.0 & 1044.9 & 1031 & 13.9 \\
\hline Bone $\left(\mathrm{CB} 230 \% \mathrm{CaCO}_{3}\right)$ & 1.340 & 1432 & 1439 & 7.4 & 1285.5 & 1252 & 33.5 \\
\hline Bone $\left(\mathrm{CB} 250 \% \mathrm{CaCO}_{3}\right)$ & 1.560 & 1770 & 1790 & 20.2 & 1469.9 & 1442 & 27.9 \\
\hline Bone mineral (B200) & 1.145 & 1347 & 1226 & 121.4 & 1094.3 & 1100 & 5.7 \\
\hline Aluminum & 2.690 & 2818 & - & - & 2335.1 & 2225 & 110.1 \\
\hline Copper & 8.849 & 39324 & - & - & 7356.5 & 6635 & 721.5 \\
\hline Titanium & 4.519 & 9638 & - & - & 3738.9 & 3383 & 355.9 \\
\hline
\end{tabular}

Phys Med Biol. Author manuscript; available in PMC 2014 August 04. 
Table 2

Treatment planning parameters for the KVCT- and MVCT-based treatment plans including gantry angle $(\theta)$, target depth $(d)$, distal margin size $(m)$, planned beam penetration range $(R)$ and smear radius $\left(r_{\mathrm{s}}\right)$. These values convey the major treatment techniques used. See the text for differences in the true dose distributions.

\begin{tabular}{llllll}
\hline \multicolumn{5}{c}{ Parameter } \\
\cline { 2 - 6 } Field orientation & $\theta\left({ }^{\circ}\right)$ & $\boldsymbol{D}(\mathbf{c m})$ & $\boldsymbol{m}(\mathbf{c m})$ & $\boldsymbol{R}(\mathbf{c m})$ & $\boldsymbol{r}_{\mathbf{s}}(\mathbf{c m})$ \\
\hline \multicolumn{5}{c}{$k V / M V$ hybrid plan } \\
Right posterior oblique & 230 & 21.30 & 1.1 & 22.3 & 0.8 \\
Left lateral & 90 & 22.90 & 1.2 & 24.0 & 0.8 \\
\multicolumn{7}{c}{$k V$ plan, with artifact correction } \\
Right posterior oblique & 230 & 21.1 & 1.1 & 22.10 & 0.8 \\
Left lateral & 90 & 24.3 & 1.2 & 24.50 & 0.9 \\
\multicolumn{7}{c}{$k V$ plan, without artifact correction } & \\
Right posterior oblique & 230 & 20.7 & 1.1 & 21.70 & 0.8 \\
Left lateral & 90 & 23.5 & 1.2 & 24.50 & 0.9 \\
\hline
\end{tabular}

